\title{
El mercado del cobre a nivel mundial: evolución, riesgos, características y potencialidades futuras
}

\author{
The copper market worldwide: trends, risks, characteristics \\ and potential future growth
}

Manuel J. Donoso Muñoz ${ }^{1}$

Recibido 30 de agosto de 2011, aceptado 30 de abril de 2013

Received: August 30, $2011 \quad$ Accepted: April 30, 2013

\begin{abstract}
RESUMEN
Este artículo examina la importancia del cobre, sobre la base de las características particulares del metal, su evolución, los riesgos y el mercado que se destaca como uno de los mercados de mayor relevancia en la industrialización de los países desarrollados y en vías de desarrollo. La minería del cobre es una de las actividades que ha venido en constante crecimiento en los últimos años previos a la crisis financiera. Los países productores y exportadores del commodity han generado un importante aporte a la economía interna que les ha permitido mitigar los efectos de la contracción económica mundial.
\end{abstract}

Palabras clave: Mercado del cobre, procesos productivos del cobre, riesgos actividad minera, producción mundial del cobre, consumo del cobre.

\section{ABSTRACT}

This article examines the importance of copper, on the base of the particular characteristics of the metal, its evolution, the risks and the market that stands out as one of the markets of greater relevance in the industrialization of the developed and developing countries. Copper mining is one of the activities that have shown constant growth in the last years, previous to the financial crisis. The producing and exporting countries of this commodity have generated an important contribution to their internal economies that has allowed them to mitigate the effects of world-wide the economic contraction.

Keywords: Market of copper, productive processes of copper, mining activity risks, world-wide production of copper, consumption of copper.

\section{INTRODUCCIÓN}

La importancia del sector minero, en particular la minería del cobre, en el desarrollo económico de los países productores ha sido y seguirá siendo de vital importancia para mejorar las condiciones de vida y el bienestar de las personas. A través del tiempo los países exportadores de este metal (principalmente Chile) han demostrado que es posible crecer a partir de la explotación de sus riquezas mineras tal es el caso de Perú, Canadá, Australia, Estados Unidos,
Suecia, Finlandia y Nueva Zelanda, entre otros. La importancia estratégica de la industria del cobre, la gran magnitud de las inversiones realizadas y la cantidad creciente de proyectos que giran en torno a esta actividad obliga a los principales agentes a la realización de estudios que permitan a las empresas mejorar permanentemente sus sistemas productivos, comerciales y de financiación, de manera de mantener un adecuado equilibrio entre los aspectos coyunturales y los estructurales de la actividad. En lo específico, la exportación de cobre

1 Universidad de Tarapacá. Escuela Universitaria de Administración y Negocios.18 de Septiembre 2222. Arica, Chile.

E-mail:mdonoso@uta.cl 
ha sido para algunos países, como el caso de Chile, un factor moderador de la reciente crisis financiera internacional.

En Chile, la industria extractiva del cobre es la principal industria, las exportaciones del metal lideran el sector exportador y en la actualidad operan las principales empresas productoras del mundo, destacando a las dos empresas con mayor producción mundial: Codelco de propiedad del Estado de Chile como la empresa de mayor producción en el mundo, seguida por la minera Escondida (de propiedad de la empresa "BHP Billinton"), siendo esta la mayor empresa privada del sector en el país.

Por otra parte, el escenario económico mundial ha puesto a los países productores frente a nuevos retos y desafíos en el sector de la minería. En lo específico, la actividad minera del cobre por tratarse de una industria a largo plazo requiere de mucha estabilidad para asegurar resultados positivos en los diferentes ciclos productivos. $\mathrm{Si}$ bien es cierto, los últimos años han evidenciado un comportamiento positivo en esta actividad, esto ha sido en gran medida consecuencia del crecimiento de economías emergentes, principalmente la de China, cuyo aumento en la actividad industrial ha tenido un impacto favorable en la exportación de materias primas para un número importante de países productores.

Para dimensionar la importancia de la minería del cobre en la actividad económica mundial en el contexto de la crisis mundial se toma como referencia los años 2006 al 2008 en que la producción y venta alcanzó un crecimiento relevante respecto de los años anteriores. En los apartados siguientes se describen los principales aspectos y antecedentes que tienen relación con el mercado del cobre. El apartado dos entrega una visión respecto de las características del cobre y una breve descripción de los principales procesos que se utilizan para obtener el metal. El apartado tres hace referencia a los riesgos que enfrenta la actividad minera del cobre. El apartado cuatro sitúa a la minería del cobre en el contexto mundial con relación a la demanda y oferta de cobre y las potencialidades futuras de este sector y el apartado cinco presenta las principales conclusiones de cada uno de los aspectos que han sido presentados en cuanto a la evolución del cobre a nivel mundial.

\section{EL COBRE Y LOS PROCESOS PRODUCTIVOS UTILIZADOS PARA SU OBTENCIÓN}

El cobre es un metal noble, no se oxida, no se degrada, no contamina y es reciclable indefinidamente. Este metal recibe las siguientes denominaciones, según el idioma que lo describa: cuprum (latín); bakar (croata); cuivre (francés); rama (italiano); kobber (noruego); kupfer (alemán); cupru (rumano); kupari (finlandés); copper (inglés) y cobre (español y portugués). Entre las principales propiedades del cobre se pueden destacar las siguientes: alta conductividad eléctrica, alto grado de conductividad térmica, excelente metal para procesos de maquinado (fácil de moldear y cortar), gran resistencia a la corrosión, alta capacidad de aleación metálica, muy buena capacidad de deformación en caliente y en frío, mantiene sus propiedades indefinidamente en el reciclaje y tiene propiedades bactericidas.

El comercio formal de este metal se hace por intermedio de la Bolsa de Metales de Londres en dólares y en lotes de 25 toneladas; el COMEX (Divisions New York Commodities Exchange) de la Bolsa Mercantil de Nueva York cuyos negocios se hacen sobre la base de lotes de 25.000 libras cotizados en centavos de dólar y la Bolsa de Metales de Shanghai donde se comercia en lotes de cinco toneladas cotizados en renminbi.

El cobre es denominado comúnmente "metal rojo" por su color rojo intenso y atractivo y su utilización puede ser de forma pura o en aleación con otros metales. En forma pura sirve para fabricar una amplia gama de productos como cables y tuberías, en forma de aleación el cobre es empleado en diversos usos industriales combinado con otros metales (las propiedades del cobre son transmitidas a las aleaciones que lo utilizan), como zinc, estaño, plata, plomo, sílice, berilio, hierro, aluminio, entre otros metales. Las dos aleaciones más importantes conocidas desde la antigüedad son el bronce (combinación cobre-estaño) y el latón (combinación cobre-zinc).

El cobre, tal como se indicó anteriormente, presenta propiedades que lo hacen muy utilizable en varias industrias, pero en especial en industrias emergentes y de alto impacto (por ejemplo, atendiendo a su alta conductividad térmica y eléctrica y su nulo 
magnetismo, el cobre es ideal para la transmisión de datos, sonidos e imágenes en el área de las telecomunicaciones).

La producción del cobre deja algunos subproductos que pueden ser comercializados en forma separada. En el caso de Chile, el principal subproducto que se obtiene de la producción del cobre es el molibdeno, este ha venido teniendo gran importancia comercial en los últimos años por cuanto es un elemento metálico utilizado como materia prima para aceros especiales y algunas otras aleaciones a las cuales aporta resistencia a la temperatura y a la corrosión, durabilidad y fortaleza.

La obtención del cobre es compleja y requiere de una serie de procesos para dejarlo en condiciones de ser comercializado, esto porque a diferencia de otras materias primas que se encuentran concentradas -como el caso del petróleo, por ejemplo-, el cobre se encuentra de manera dispersa en grandes áreas, mezclado con una gran cantidad de sales y otros elementos mineralógicos además de roca estéril. Estos compuestos que contienen cobre deben ser tratados debidamente para separar el metal del resto de los minerales y se clasifican en dos grupos de acuerdo con su composición química: (a) minerales sulfurados (con alto contenido de azufre) y (b) minerales oxidados (con elevado contenido de óxido de carbono). Los minerales sulfurados más representativos son: malaquita, calcantita, atacamita y cuprita. Por su parte, los minerales oxidados más representativos son: calcosina, calcopirita y bornita.

El porcentaje de cobre presente en estos minerales es conocido por los especialistas como "Ley del cobre" y su valor es variable (por ejemplo, si la ley fuese $1,2 \%$ significa que por cada mil kilos o tonelada métrica de mineral hay 12 kilos de cobre fino). En los yacimientos mineros de Chile esa ley fluctúa de 1 a 1,8 por ciento según los estudios geológicos que la determinan. Cuanto menor es la ley, menor es la cantidad de cobre contenido en el material mineralógico explotado en las minas, por esta razón gran parte de este material constituye desecho y se acumula en lugares especiales denominados botaderos una vez que el cobre ha sido debidamente separado.

La dispersión con que aparece el cobre hace necesario someter los minerales extraídos (sean estos sulfurados o minerales oxidados) a procesos productivos con el fin de obtener un metal puro. Estos dos tipos de minerales requieren de procesos productivos diferentes, pero en ambos casos, el punto de partida es el mismo, es decir, la extracción del material mineralógico desde las minas a cielo (rajo) abierto (minas que se operan en la superficie) o subterráneas (minas que se operan en túneles subterráneos), lo que requiere la fragmentación (reducción de su tamaño) y el transporte del material mineralógico identificado en los estudios geológicos realizados en la etapa previa de exploración.

El material mineralógico extraído pasa por diferentes procesos según el tipo de mineral de que se trate. En el caso de los minerales oxidados el proceso productivo implica someter el material a un proceso inicial de molienda y posteriormente a un proceso de lixiviación (proceso hidrometalúrgico), para producir soluciones de sulfato de cobre a través de una solución acuosa compuesta de ácido sulfúrico y agua (esta solución disuelve el cobre contenido en los minerales oxidados). Posteriormente, el sulfato de cobre es separado de las impurezas mediante un proceso de extracción con solventes (productos químicos), proceso que entrega el concentrado de cobre. La posibilidad de alcanzar cobre con mayor grado de pureza se realiza por un proceso electrometalúrgico denominado "electroobtención", que consiste en utilizar celdas electrolíticas (uso de corriente eléctrica en solución acuosa) para purificar el cobre concentrado, es decir, eliminar las impurezas (soluciones químicas) que provienen de los procesos anteriores, cuyo resultado final son los cátodos de cobre $\mathrm{SxEw}$ (donde $\mathrm{Sx}=$ extracción por solventes y Ew $=$ refinado vía proceso electrometalúrgico) con 99,99 por ciento de pureza.

Por su parte, los minerales sulfurados pasan primero por el proceso de trituración (denominado comúnmente "chancado") y molienda para luego ser clasificados (separar aquellos minerales que han alcanzado una dimensión adecuada) y continuar el proceso de concentrado (este proceso consta de dos procesos intermedios, el de flotación, que es un proceso físico-químico que permite a través de una solución acuosa separar los minerales sulfurados que contienen cobre de aquel material que no lo contiene y el de secado que consiste en separar el cobre mediante un proceso de decantación y secarlo hasta obtener cobre concentrado que tiene aproximadamente un 30 por ciento del metal). 


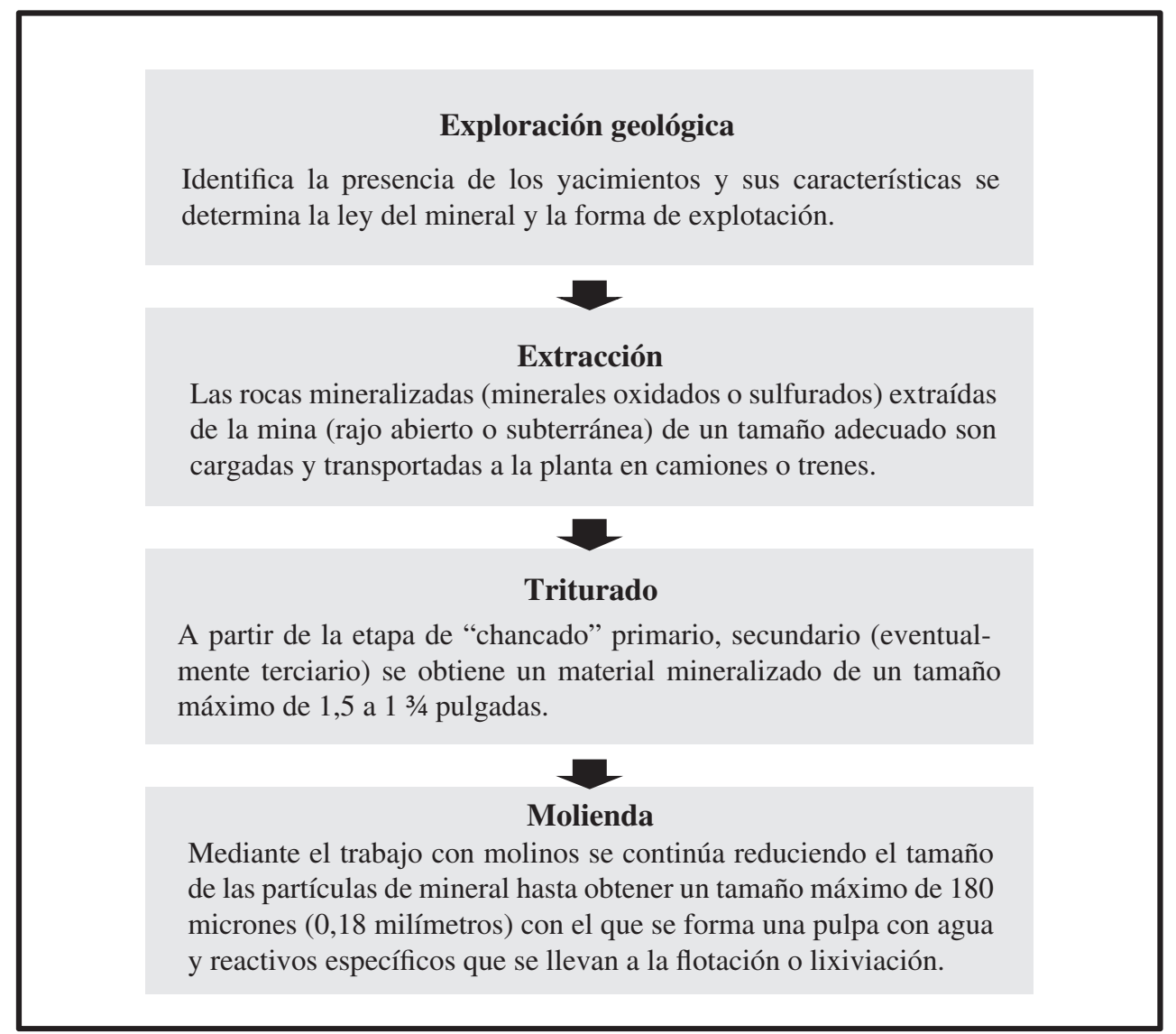

Figura 1. Proceso productivo común: minerales oxidados y sulfurados.

Su purificación posterior se realiza en hornos especiales (hornos de refino blíster) que permiten obtener cobre blíster (láminas de cobre) con 96 por ciento de pureza. El cobre blíster puede ser vendido directamente o continuar un proceso de mayor refinamiento. El proceso de refinado siguiente puede ser a fuego hasta obtener cobre RAF (refinado a fuego), cobre con 99,7 por ciento de pureza o seguir un camino alternativo de refinación mediante moldeo en ánodos (hornos especiales que utilizan placas moldeadoras llamadas ánodos) y que una vez completado el proceso de fundición el cobre es purificado por un proceso electrometalúrgico denominado "electrorrefinación" que consiste en utilizar celdas electrolíticas (uso de corriente eléctrica en solución acuosa) para purificar las placas hasta obtener cátodos ER (electrorrefinado) con un 99,99 por ciento de pureza. Los procesos descritos se muestran en las Figuras 1, 2 y 3 respectivamente.

\section{LOS RIESGOS ASOCIADOS A LA ACTIVIDAD MINERA DEL COBRE}

Un aspecto relevante en la actividad minera del cobre, al igual que otras actividades similares, es la presencia permanente de una serie de factores de riesgo que obligan a las empresas a gestionarlos debidamente para evitar un impacto negativo en sus resultados [1]. A continuación se describen algunos de los riesgos más relevantes que afectan a la actividad:

\section{Riesgos de la Unidad Estratégica de negocio}

Son aquellos que la empresa asume con el fin de obtener una ventaja competitiva y crear valor sostenido para los accionistas. Algunos autores utilizan la expresión Riesgo económico o de la empresa para denotarlo. En cualquier caso, se trata de riesgos inherentes al par producto-mercado, como 


\section{Pilas de lixiviación}

La lixiviación en pilas (piscinas) es un proceso hidrometalúrgico (obtención de metales a partir de reacciones que tienen lugar en fases acuosas) que permite obtener cobre de los minerales oxidados que lo contienen, aplicando una solución de ácido sulfúrico y agua para disolver el cobre y transformarlo en una solución denominada "sulfato de cobre" que es recogida por un sistema de drenaje ubicado en la parte inferior de las pilas.

\section{Extracción por solvente}

En esta etapa la solución que viene de las pilas de lixiviación se separa de las impurezas que pueda haber arrastrado para concentrar su contenido de cobre (extracción iónica).

\section{Electroobtención}

Aquí la solución electrolítica de cobre es llevada a las celdas de electroobtención que tienen sumergidas en su interior unas placas metálicas (un ánodo o placas de plomo que actúa como polo positivo y un cátodo o placas de acero que actúa como polo negativo). Así se recupera el cobre obteniéndose cátodos de alta pureza.

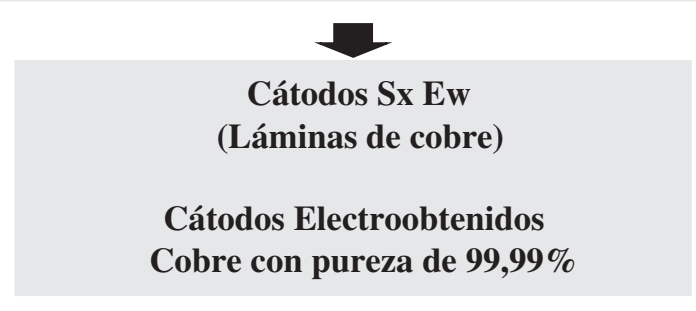

Figura 2. Proceso productivo minerales oxidados.

por ejemplo, las innovaciones tecnológicas, el diseño y lanzamiento de un nuevo producto o servicio, el marketing mix, etcétera. Sirva de ejemplo el comercio electrónico o comercio vía Internet, que, en el caso de la minería actualmente se configura como una nueva forma de comercialización de los metales.

\section{Riesgos Estratégicos}

Hacen referencia a cambios o acontecimientos inesperados en la economía o en el contexto político de ciertos países que pueden afectar la actividad empresarial mundial de las empresas productoras del metal (por ejemplo, la guerra de Irak, los cambios de enfoque en los gobiernos en América del Sur, el crecimiento económico de China, entre otros acontecimientos importantes).

\section{Riesgos Financieros}

Son aquellos que aparecen en los mercados financieros y se traducen, generalmente, en importantes pérdidas de capital. Los riesgos financieros a su vez también se pueden clasificar a partir de su relación con determinadas variables financieras [2]. A continuación se presentan y enumeran los riesgos financieros más relevantes:

Riesgos operacionales: Son aquellos que se originan a partir de ciertos aspectos propios de las 


\section{Flotación}

En las celdas de flotación se genera espuma cuyas burbujas arrastran el cobre y otros minerales contenidos en la pulpa, luego de varios ciclos se recolecta y seca la espuma para obtener el concentrado de cobre que continúa su purificación.

\section{Fundición}

Para separar el cobre de otros minerales e impurezas el concentrado de cobre seco se trata a grandes temperaturas en hornos especiales (hornos blíster), luego de varios procesos (recepción y muestreo, fusión y conversión) se obtiene cobre en láminas, denominado blíster.

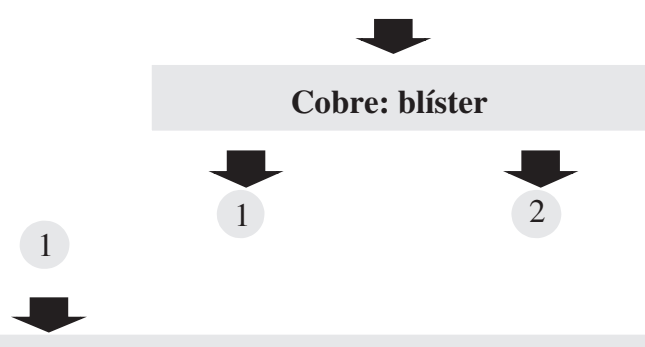

\section{Pirorrefinación}

Refinación del cobre blíster eliminando el porcentaje de oxígeno existente hasta obtener cobre refinado y moldeado en placas gruesas.

\section{Cobre RAF \\ Refinado a fuego \\ Cobre con pureza de $\mathbf{9 9 , 7 \%}$}

\section{Electrorrefinación}

Los ánodos provenientes de la fundición se llevan a celdas electrolíticas para su refinación.

De este proceso se obtienen cátodos de alta pureza (cátodos de cobre electrolítico) de 70 y $80 \mathrm{~kg}$.

\section{Cátodos ER:}

Cátodos electrorrefinados

Cobre con pureza de $\mathbf{9 9 , 9 9 \%}$

Figura 3. Proceso productivo minerales sulfurados. 
operaciones. Entre los más conocidos tenemos los que se generan por anomalías en la infraestructura tecnológica (Riesgo tecnológico); errores humanos en la ejecución de procesos (Riesgo de ejecución) o de información fraudulenta (Riesgo de fraude) respecto de una operación financiera. Igualmente aparece cuando se utiliza un modelo inadecuado para valorar una posición de mercado (Riesgo de modelo).

Riesgo de liquidez: Pérdidas que se producen ante la existencia de una contrapartida para deshacer una posición de mercado (Riesgo de liquidez en mercado o producto), este tipo de riesgo también es llamado Riesgo de contratación. Es particularmente característico en mercados de oferta y demanda directas, como son los mercados organizados (OTC). Cuando el Riesgo de liquidez se deriva de la dificultad para hacer frente a obligaciones de pago futuras se denomina Riesgo de liquidez en flujo de caja.

Riesgo legal: Este tipo de riesgo se manifiesta cuando una modificación legal o cambia de manera significativa los términos establecidos originalmente en una transacción o cuando existe un vacío legal o no hay jurisprudencia que apoye una posición. Este tipo de riesgo también emerge cuando una de las partes carece de autoridad legal suficiente para llevar a cabo una transacción económica.

Riesgo de crédito: Surge frente a la posibilidad de incumplimiento de las obligaciones contractuales entre las partes de una transacción financiera. Este tipo de riesgo también aparece cuando las agencias de calificación deciden modificar la calidad crediticia del deudor.

Riesgo de mercado: Denominado comúnmente Riesgo de posición, representa un cambio en variables como precio del commodity, tipo de interés y tipo de cambio sobre posiciones abiertas en los mercados financieros que pueden incidir de manera significativa en el valor de la posición mantenida por un operador de una empresa o entidad financiera.

\section{Riesgos del proyecto minero}

La errónea estimación de los costos operacionales en la determinación de la ley del mineral mediante las pruebas metalúrgicas (aquellas que estiman la cantidad de cobre contenido en cada tonelada de mineral) y en la obtención de las muestras empleadas para hacerlas y/o la subestimación de la inversión en la etapa de evaluación, pueden condicionar el desarrollo futuro del proyecto minero.

\section{Riesgo de la naturaleza}

La sobrevaloración de la calidad del yacimiento minero puede en su etapa de operación dar lugar a factores adversos, como terremotos, deslizamientos, fallas geológicas, entre otros similares.

\section{LA MINERÍA DEL COBRE A NIVEL MUNDIAL}

Si bien es cierto que la industria minera es relativamente pequeña en el contexto global de todas las actividades económicas (un estudio realizado por la Comisión Chilena del Cobre, durante el año 2004 [3] entregó como antecedentes que, tomando en cuenta la capitalización de mercado de todas las empresas que transan en el mercado norteamericano -acciones o $\mathrm{ADR}^{2}$ - que alcanza a US $\$ 2$ billones, la del sector minería metálica corresponde a US\$ 328 mil millones, cifra que representa solo el $1,15 \%$ de la capitalización total y sumado el sector de la minería no metálica aumenta a US\$ $467 \mathrm{mil}$ millones alcanzando el 2,2\% de la capitalización total), la situación hacia el año 2006 y siguientes ha cambiado, otorgándole al sector minero una mayor relevancia, esto principalmente, por la irrupción comercial e industrial de nuevas economías del Asia Pacífico y el fortalecimiento económico de países de Europa del Este y también, aunque en menor grado, de América latina. Por ejemplo, en la Bolsa de Valores de Londres (LSE) el sector minero en su totalidad alcanzó aproximadamente el 4,1\% de la capitalización total (cerca de US\$210 mil millones) en el primer semestre del año 2007.

En efecto, la industria minera no ha sido muy rentable en las últimas décadas y solo se aprecia un repunte en los últimos años, con precios crecientes a partir de 2003. Si se comparan entre 1977 y 1999, las rentabilidades bursátiles ${ }^{3}$ de la industria de metales básicos con los bonos de gobierno de los Estados Unidos con vencimiento a 10 años, se observa que durante muchos años la rentabilidad de la industria fue mucho menor que la tasa libre

\footnotetext{
2 ADR (American Depositary Receipt) es una acción de una corporación extranjera que puede comprarse o venderse en el mercado de los Estados Unidos.

3 Precio de acciones y dividendos.
} 
de riesgo (rentabilidad de los bonos). Incluso en ese periodo la rentabilidad promedio de la industria minera completa $(5,7 \%)$ fue menor al de los bonos $(6,7 \%)^{4}[4]$.

El pobre desempeño en las décadas pasadas se debió al exceso de oferta con relación a la demanda existente a nivel mundial generando una baja general de los precios en los mercados. Esta situación ha cambiado con la aparición de economías emergentes que han incrementado la demanda sobre distintos tipos de metales, en especial el cobre, reduciendo los niveles de inventarios, aumentando la producción y mejorando el precio del commodity.

El aumento en la demanda con el consiguiente incremento en los precios del cobre ha permitido que se reactiven proyectos mineros que parecían destinados al fracaso, esto ha constituido una fuerte inversión en nuevas tecnologías y en investigación y desarrollo. Chile es un buen ejemplo del nuevo impulso que está teniendo la actividad minera.

\section{Demanda y consumo mundial}

El informe de la Comisión Chilena del Cobre [5] destaca algunos aspectos de la demanda que conviene precisar, con relación a los países consumidores de cobre: La irrupción de China en la economía mundial atrae especial atención, no solo por su sobresaliente crecimiento, en torno al 10\% durante los últimos años, sino que también por su enorme tamaño. En la actualidad China es la cuarta economía del mundo, medido a paridad del poder de compra, y contribuyó en un $25 \%$ del crecimiento mundial a partir de 2006. La aparición de China en la economía mundial ha traído consigo efectos positivos y negativos para los países productores de productos básicos. Para aquellos países en los que una fracción importante de sus exportaciones corresponde a manufacturas China ha pasado a ser un competidor importante, como es el caso de México y especialmente Brasil en América latina [6]. En cambio, aquellos que exportan principalmente commodities (ejemplo, Chile) han visto incrementada su demanda externa. El deterioro del sector viviendas en EE.UU. que dio origen a la crisis financiera mundial, ha influido en la demanda de cobre corrigiéndose a la baja a partir de 2007. La demanda comunitaria de cobre de la Unión Europea ha tenido una caída cercana al 4,6\%

4 Fuente: "Mining in the global market", 2001. P. Crowson. en 2007 y un porcentaje mayor durante 2008, luego de haber tenido un robusto crecimiento de $11 \%$ en 2006, similar situación se ha dado para Japón que luego de crecer un 4,8\% en 2006 cayó durante 2007 en un 2,7\% [7]. Las Tablas 1 y 2 muestran la demanda de cobre refinado medida por el flujo de importaciones de toneladas métricas del metal y el consumo mundial.

\section{Oferta y producción mundial}

La oferta de cobre ha venido sosteniendo un incremento importante durante los últimos años en sus distintas formas de explotación (producción de cobre mina, procesado en fundiciones o cobre debidamente refinado: cátodos $\mathrm{Sx}-\mathrm{Ew}$, cátodos E.R. o refinado a fuego). La oferta final materializada de acuerdo con las necesidades de los países consumidores se puede verificar mediante las exportaciones del metal que los distintos países productores han tenido en los últimos años. Durante 2007 Chile tuvo una participación en el total de exportaciones del metal de 40,4\% con una leve caída en 2008 alcanzando el $39,1 \%$ del total de las exportaciones mundiales. Las Tablas 3, 4, y 5 muestran la producción de cobre en sus distintas formas. La Tabla 6 incluye información complementaria que muestra la producción de cobre en cátodos $\mathrm{Sx}$-Ew (dicha producción está contenida en la producción de cobre mina que se muestra en la Tabla 3) y la Tabla 7 el total de exportaciones de cobre en el mundo. [8]

Respecto de las empresas productoras, entre los actores más relevantes de la industria del cobre en el mundo, Codelco (empresa de propiedad del Estado chileno) aparece como el principal explotador de la materia prima, con una participación cercana al $16 \%$ de la producción mundial, seguida por la australiana BHP Billiton, la minera privada más grande del mundo con un $10 \%$ de participación en la producción mundial. En tercer lugar está la estadounidense Phelps Dodge, con un 9\%. La Tabla 8 muestra las principales empresas productoras de cobre en el mundo, tomando como referencia la producción del año 2008 [9].

\section{Potencialidades y proyectos en la minería del cobre a nivel mundial}

Según la información recopilada por la Comisión Chilena del Cobre a mayo 2007 [10], existía a esa fecha una cartera de proyectos y prospectos mineros para ser ejecutados en los años siguientes 
Tabla 1. Importaciones mundiales de cobre (miles de toneladas métricas).

\begin{tabular}{|l|r|r|r|r|}
\hline \multicolumn{1}{|c|}{ País /Año } & \multicolumn{1}{c|}{$\mathbf{2 0 0 0}$} & $\mathbf{2 0 0 6}$ & \multicolumn{1}{c|}{$\mathbf{2 0 0 7}$} & \multicolumn{1}{c|}{$\mathbf{2 0 0 8}$} \\
\hline China & $1.245,7$ & $1.823,5$ & $2.800,8$ & $2.955,1$ \\
\hline Japón & $1.570,4$ & $1.411,4$ & $1.531,3$ & $1.443,0$ \\
\hline Alemania & 899,2 & $1.210,1$ & $1.200,6$ & $1.130,1$ \\
\hline Estados Unidos & $1.216,4$ & $1.248,9$ & 997,9 & 908,2 \\
\hline Corea del Sur & 761,2 & 831,9 & 870,6 & 835,7 \\
\hline Italia & 606,0 & 774,5 & 747,3 & 617,8 \\
\hline Taiwán & 629,0 & 646,6 & 614,9 & 585,0 \\
\hline Francia & 585,9 & 507,0 & 432,2 & 434,1 \\
\hline India & 128,2 & 617,9 & 538,3 & 411,9 \\
\hline Brasil & 279,5 & 309,5 & 338,7 & 370,3 \\
\hline España & 282,5 & 340,7 & 307,6 & 363,4 \\
\hline Principales países & $\mathbf{8 . 2 0 4 , 0}$ & $\mathbf{9 . 7 2 2 , 0}$ & $\mathbf{1 0 . 3 8 0 , 2}$ & $\mathbf{1 0 . 0 5 4 , 6}$ \\
\hline Resto del mundo & $2.723,9$ & $2.922,9$ & $2.519,2$ & $2.494,9$ \\
\hline Total mundial & $\mathbf{1 0 . 9 2 7 , 9}$ & $\mathbf{1 2 . 6 4 4 , 9}$ & $\mathbf{1 2 . 8 9 9 , 4}$ & $\mathbf{1 2 . 5 4 9 , 5}$ \\
\hline
\end{tabular}

Fuente: Comisión Chilena del Cobre (Cochilco).

Tabla 2. Consumo mundial de cobre refinado (miles de toneladas métricas de cobre fino).

\begin{tabular}{|l|r|r|r|r|}
\hline \multicolumn{1}{|c|}{ País /Año } & \multicolumn{1}{c|}{$\mathbf{2 0 0 0}$} & \multicolumn{1}{c|}{$\mathbf{2 0 0 6}$} & \multicolumn{1}{c|}{$\mathbf{2 0 0 7}$} & \multicolumn{1}{c|}{$\mathbf{2 0 0 8}$} \\
\hline China & \multicolumn{1}{c|}{$1.928,1$} & $3.613,8$ & $4.863,4$ & \multicolumn{1}{c|}{$5.133,6$} \\
\hline Estados Unidos & $3.025,5$ & $2.096,0$ & $2.140,0$ & $1.913,6$ \\
\hline Alemania & $1.307,1$ & $1.397,6$ & $1.391,8$ & $1.386,5$ \\
\hline Japón & $1.349,2$ & $1.282,3$ & $1.251,9$ & $1.184,4$ \\
\hline Corea del Sur & 862,2 & 827,9 & 857,6 & 851,6 \\
\hline Rusia & 183,0 & 693,3 & 687,8 & 730,8 \\
\hline Italia & 673,9 & 800,5 & 763,6 & 638,2 \\
\hline Taiwán & 628,4 & 642,5 & 603,0 & 582,3 \\
\hline India & 240,2 & 406,7 & 516,1 & 514,6 \\
\hline Principales países & $\mathbf{1 0 . 1 9 7 , 6}$ & $\mathbf{1 1 . 7 6 0 , 6}$ & $\mathbf{1 3 . 0 7 5 , 2}$ & $\mathbf{1 2 . 9 3 5 , 6}$ \\
\hline Resto del mundo & $4.994,3$ & $5.213,8$ & $5.019,2$ & $5.088,6$ \\
\hline Total mundial & $\mathbf{1 5 . 1 9 1 , 9}$ & $\mathbf{1 6 . 9 7 4 , 4}$ & $\mathbf{1 8 . 0 9 4 , 4}$ & $\mathbf{1 8 . 0 2 4 , 2}$ \\
\hline
\end{tabular}

Fuente: Comisión Chilena del Cobre (Cochilco).

por las empresas cupríferas, información que permite formarse una idea respecto del volumen de inversiones comprometidas en este sector, así también cuantificar las reservas, leyes del mineral involucradas (porcentaje de cobre que se obtiene por cada tonelada de mineral extraído) y la producción real y potencial para el período comprendido entre 2006 y 2015.

Para estos efectos se define como "proyectos" cupríferos aquellos desarrollos de yacimientos en que existen suficientes elementos que permiten cuantificar adecuadamente las reservas (en miles de toneladas métricas), la ley media de contenido de cobre y de otros elementos (en porcentaje de contenido del metal por cada tonelada de mineral extraído), inversión (en miles de dólares), producción nueva involucrada (toneladas métricas finales). Por su parte, la denominación de "prospecto" cuprífero corresponde a todos aquellos proyectos en formación (desarrollos de yacimientos que no cuentan con información en uno o varios de los aspectos señalados en el caso de los proyectos y que se encuentran en una etapa bastante preliminar, generalmente en la etapa de cuantificación de reservas).

Según los estudios de la Comisión Chilena del Cobre ${ }^{5}$ [11], el resumen de los proyectos mineros de cobre informados en el periodo señalado precedentemente corresponde a 166 proyectos mineros de cobre, de

5 Documento preparado por Aldo Picozzi, 2007. 
Tabla 3. Producción de cobre mina* (miles de toneladas métricas en cobre fino).

\begin{tabular}{|l|r|r|r|r|}
\hline \multicolumn{1}{|c|}{ País /Año } & $\mathbf{2 0 0 0}$ & $\mathbf{2 0 0 6}$ & \multicolumn{1}{c|}{$\mathbf{2 0 0 7}$} & \multicolumn{1}{c|}{$\mathbf{2 0 0 8}$} \\
\hline Chile & $4.602,0$ & $5.360,8$ & $5.557,0$ & \multicolumn{1}{c|}{$5.330,3$} \\
\hline Estados Unidos & $1.444,1$ & $1.197,0$ & $1.190,0$ & $1.339,7$ \\
\hline Perú & 553,9 & $1.048,5$ & $1.190,3$ & $1.267,9$ \\
\hline China & 592,6 & 872,9 & 928,0 & 930,8 \\
\hline Australia & 832,0 & 875,0 & 871,0 & 885,0 \\
\hline Rusia & 580,0 & 779,4 & 770,0 & 784,8 \\
\hline Indonesia & $1.005,5$ & 816,6 & 788,9 & 650,5 \\
\hline Canadá & 639,9 & 603,3 & 596,2 & 607,0 \\
\hline Zambia & 249,3 & 515,6 & 550,3 & 595,0 \\
\hline Polonia & 454,1 & 497,1 & 452,0 & 436,0 \\
\hline Kazajstán & 430,2 & 446,3 & 406,5 & 419,9 \\
\hline Principales países & $\mathbf{1 1 . 3 8 3 , 6}$ & $\mathbf{1 3 . 0 1 2 , 5}$ & $\mathbf{1 3 . 3 0 0 , 2}$ & $\mathbf{1 3 . 2 4 6 , 9}$ \\
\hline Resto del mundo & $1.862,9$ & $2.197,9$ & $2.349,7$ & $2.344,1$ \\
\hline Total mundial & $\mathbf{1 3 . 2 4 6 , 5}$ & $\mathbf{1 5 . 2 1 0 , 4}$ & $\mathbf{1 5 . 6 4 9 , 9}$ & $\mathbf{1 5 . 5 9 1 , 0}$ \\
\hline \% Participación Chile & $\mathbf{3 4 , 7}$ & $\mathbf{3 5 , 2}$ & $\mathbf{3 5 , 5}$ & $\mathbf{3 4 , 2}$ \\
\hline
\end{tabular}

Fuente: Comisión Chilena del Cobre (Cochilco).

*Producción que incluye cobre concentrado con 93\% de pureza y cobre en cátodos SxEw con 99,99\% de pureza (el detalle de la producción de cobre refinado en cátodos SxEw se muestra en la Tabla 6).

Tabla 4. Producción de cobre fundición* (miles de toneladas métricas en cobre fino).

\begin{tabular}{|l|r|r|r|r|}
\hline \multicolumn{1}{|c|}{ País /Año } & \multicolumn{1}{c|}{$\mathbf{2 0 0 0}$} & \multicolumn{1}{c|}{$\mathbf{2 0 0 6}$} & \multicolumn{1}{c|}{$\mathbf{2 0 0 7}$} & \multicolumn{1}{c|}{$\mathbf{2 0 0 8}$} \\
\hline China & $1.013,9$ & $1.917,5$ & $2.111,5$ & $2.495,5$ \\
\hline Japón & $1.480,6$ & $1.628,3$ & $1.612,5$ & $1.625,4$ \\
\hline Chile & $1.460,4$ & $1.565,4$ & $1.514,3$ & $1.369,2$ \\
\hline Rusia & 758,0 & 850,0 & 850,0 & 850,0 \\
\hline India & 225,6 & 629,0 & 713,9 & 660,9 \\
\hline Estados Unidos & $1.002,0$ & 500,8 & 617,0 & 550,1 \\
\hline Alemania & 571,6 & 510,0 & 510,0 & 510,0 \\
\hline Canadá & 603,7 & 520,5 & 516,8 & 487,1 \\
\hline Polonia & 482,5 & 589,4 & 558,6 & 470,8 \\
\hline Australia & 386,9 & 377,0 & 399,0 & 447,0 \\
\hline Kazajstán & 414,9 & 428,4 & 392,8 & 392, \\
\hline Principales países & $\mathbf{8 . 4 0 0 , 1}$ & $\mathbf{9 . 5 1 6 , 3}$ & $\mathbf{9 . 7 9 6 , 4}$ & $\mathbf{9 . 8 5 8 , 3}$ \\
\hline Resto del mundo & $3.455,3$ & $3.787,7$ & $3.611,1$ & $3.748,5$ \\
\hline Total mundial & $\mathbf{1 1 . 8 5 5 , 4}$ & $\mathbf{1 3 . 3 0 4 , 0}$ & $\mathbf{1 3 . 4 0 7 , 5}$ & $\mathbf{1 3 . 6 0 6 , 8}$ \\
\hline \% Participación Chile & $\mathbf{1 2 , 3}$ & $\mathbf{1 1 , 8}$ & $\mathbf{1 1 , 3}$ & $\mathbf{1 0 , 1}$ \\
\hline
\end{tabular}

Fuente: Comisión Chilena del Cobre (Cochilco).

* Producción de cobre blister (cobre con 96\% de pureza).

los cuales 139 son nuevos (proyectos greenfield ${ }^{6}$ ) y 27 son proyectos de ampliación de capacidad de producción o reinicio de operaciones, asociados a minas que se encuentran en operación o corresponden a reaperturas de minas cerradas en años anteriores

\footnotetext{
6 Se define como proyecto greenfield aquel que se desarrolla en un yacimiento nuevo.
}

(proyectos brownfield ${ }^{7}$ ). A nivel de producto final, 111 de estos proyectos producirán concentrados de cobre $(67 \%)$ y 55 de ellos producirán cátodos electroobtenidos por la vía hidrometalúrgica. $\mathrm{La}$

7 Se define como proyecto brownfield aquel que se desarrolla en un sector de un yacimiento en operación o en un sector contiguo pero relacionado. Igualmente se define en forma similar cuando el proyecto está asociado a un yacimiento antiguo que se encuentre cerrado. 
Tabla 5. Producción de cobre refinado* (miles de toneladas métricas en cobre fino).

\begin{tabular}{|l|r|r|r|r|}
\hline \multicolumn{1}{|c|}{ País /Año } & $\mathbf{2 0 0 0}$ & $\mathbf{2 0 0 6}$ & \multicolumn{1}{c|}{$\mathbf{2 0 0 7}$} & \multicolumn{1}{c|}{$\mathbf{2 0 0 8}$} \\
\hline China & $1.371,1$ & $3.003,2$ & $3.499,4$ & $3.779,3$ \\
\hline Chile & $2.668,3$ & $2.811,3$ & $2.936,5$ & $3.060,3$ \\
\hline Japón & $1.437,4$ & $1.532,1$ & $1.576,8$ & $1.539,8$ \\
\hline Estados Unidos & $1.802,1$ & $1.250,2$ & $1.310,0$ & $1.275,0$ \\
\hline Rusia & 824,0 & 959,2 & 923,3 & 926,4 \\
\hline Alemania & 709,5 & 662,3 & 665,5 & 697,1 \\
\hline India & 259,1 & 626,8 & 718,6 & 669,2 \\
\hline Zambia & 226,2 & 497,2 & 522,0 & 604,7 \\
\hline Corea del Sur & 470,5 & 575,5 & 584,5 & 573,0 \\
\hline Polonia & 486,0 & 556,6 & 533,0 & 526,7 \\
\hline Australia & 484,0 & 429,0 & 442,0 & 503,0 \\
\hline Principales países & $\mathbf{1 0 . 7 3 8 , 2}$ & $\mathbf{1 2 . 9 0 3 , 4}$ & $\mathbf{1 3 . 7 1 1 , 6}$ & $\mathbf{1 4 . 1 5 4 , 5}$ \\
\hline Resto del mundo & $4.077,6$ & $4.439,9$ & $4.268,2$ & $4.329,1$ \\
\hline Total mundial & $\mathbf{1 4 . 8 1 5 , 8}$ & $\mathbf{1 7 . 3 4 3 , 3}$ & $\mathbf{1 7 . 9 7 9 , 8}$ & $\mathbf{1 8 . 4 8 3 , 6}$ \\
\hline \% Participación Chile & $\mathbf{1 8 , 0}$ & $\mathbf{1 6 , 2}$ & $\mathbf{1 6 , 3}$ & $\mathbf{1 6 , 6}$ \\
\hline
\end{tabular}

Fuente: Comisión Chilena del Cobre (Cochilco).

*Producción de cobre refinado: refinado a fuego (cobre RAF con pureza de 99,3\%) y refinado electrólisis (cobre en cátodos ER, con 99,99\% de pureza).

Tabla 6. Producción de Sx-Ew* (miles de toneladas métricas en cobre fino).

\begin{tabular}{|l|r|r|r|r|}
\hline \multicolumn{1}{|c|}{ País /Año } & \multicolumn{1}{c|}{$\mathbf{2 0 0 0}$} & \multicolumn{1}{c|}{$\mathbf{2 0 0 6}$} & \multicolumn{1}{c|}{$\mathbf{2 0 0 7}$} & \multicolumn{1}{c|}{$\mathbf{2 0 0 8}$} \\
\hline Chile & $1.372,3$ & $1.691,8$ & $1.832,1$ & $1.973,7$ \\
\hline Estados Unidos & 556,0 & 529,8 & 504,0 & 530,0 \\
\hline Perú & 127,3 & 173,9 & 172,1 & 160,1 \\
\hline Principales países & $\mathbf{2 . 0 5 5 , 6}$ & $\mathbf{2 . 3 9 5 , 5}$ & $\mathbf{2 . 5 0 8 , 2}$ & $\mathbf{2 . 6 6 3 , 8}$ \\
\hline Resto del mundo & 254,0 & 332,5 & 301,5 & 323,1 \\
\hline Total mundial & $\mathbf{2 . 3 0 9 , 6}$ & $\mathbf{2 . 7 2 8 , 0}$ & $\mathbf{2 . 8 0 9 , 7}$ & $\mathbf{2 . 9 8 6 , 9}$ \\
\hline \% Participación Chile & $\mathbf{5 9 , 4}$ & $\mathbf{6 2 , 0}$ & $\mathbf{6 5 , 2}$ & $\mathbf{6 6 , 1}$ \\
\hline
\end{tabular}

Fuente: Comisión Chilena del Cobre (Cochilco).

*Detalle de la producción de cobre en cátodos SxEw ya incluida en producción de cobre mina (ver Tabla 3).

Tabla 7. Exportaciones mundiales de cobre (miles de toneladas métricas).

\begin{tabular}{|l|r|r|r|r|}
\hline \multicolumn{1}{|c|}{ País /Año } & \multicolumn{1}{c|}{$\mathbf{2 0 0 0}$} & \multicolumn{1}{c|}{$\mathbf{2 0 0 6}$} & \multicolumn{1}{c|}{$\mathbf{2 0 0 7}$} & \multicolumn{1}{c|}{$\mathbf{2 0 0 8}$} \\
\hline Chile & $4.473,0$ & $5.234,0$ & $5.673,4$ & \multicolumn{1}{c|}{$5.405,3$} \\
\hline Perú & 480,3 & 927,2 & $1.100,3$ & $1.194,7$ \\
\hline Australia & 578,7 & 680,6 & 676,1 & 808,3 \\
\hline Canadá & 822,9 & 598,6 & 609,0 & 724,8 \\
\hline Zambia & 216,6 & 476,1 & 490,9 & 585,2 \\
\hline Indonesia & 775,5 & 693,6 & 600,4 & 550,0 \\
\hline Japón & 342,1 & 329,1 & 437,8 & 436,0 \\
\hline Estados Unidos & 296,3 & 525,4 & 369,9 & 365,2 \\
\hline Principales países & $\mathbf{7 . 9 8 5 , 4}$ & $\mathbf{9 . 4 6 4 , 6}$ & $\mathbf{9 . 9 5 7 , 8}$ & $\mathbf{1 0 . 0 6 9 , 5}$ \\
\hline Resto del mundo & $3.537,3$ & $4.065,5$ & $4.081,6$ & $3.754,6$ \\
\hline Total mundial & $\mathbf{1 1 . 5 2 2 , 7}$ & $\mathbf{1 3 . 5 3 0 , 1}$ & $\mathbf{1 4 . 0 3 9 , 4}$ & $\mathbf{1 3 . 8 2 4 , 1}$ \\
\hline \% Participación Chile & $\mathbf{3 8 , 8}$ & $\mathbf{3 8 , 7}$ & $\mathbf{4 0 , 4}$ & $\mathbf{3 9 , 1}$ \\
\hline
\end{tabular}

Fuente: Comisión Chilena del Cobre (Cochilco). 
Tabla 8. Principales empresas productoras de cobre en el mundo (porcentaje producción mundial).

\begin{tabular}{|l|l|l|c|}
\hline \multicolumn{1}{|c|}{ Empresa } & \multicolumn{1}{|c|}{$\begin{array}{c}\text { País* } \\
\text { inversor principal }\end{array}$} & \multicolumn{1}{c|}{$\begin{array}{c}\text { País** } \\
\text { productor }\end{array}$} & $\begin{array}{c}\text { Participación en la producción } \\
\text { mundial año 2008 }\end{array}$ \\
\hline Codelco & Chile & Chile & $16 \%$ \\
\hline BHP Billiton & Australia & Australia, Perú y Chile & $10 \%$ \\
\hline Phelps Dodge & EE.UU. & EE.UU., Chile y Perú & $9 \%$ \\
\hline Rio Tinto & Inglaterra & $\begin{array}{l}\text { EE.UU., Australia, } \\
\text { Zimbabwe y Chile }\end{array}$ & $7 \%$ \\
\hline Grupo México & México & México y Perú & $6 \%$ \\
\hline Anglo American & Inglaterra & Sudáfrica, Chile y Perú & $6 \%$ \\
\hline
\end{tabular}

Fuente: Corporación Chilena del Cobre.

* País donde se encuentra la casa matriz. ** País donde se encuentran los yacimientos mineros.

Tabla 9. Evolución del precio nominal del cobre (centavos de dólar por libra).

\begin{tabular}{|c|c|c|c|}
\hline Año & $\begin{array}{c}\text { Precio promedio anual } \\
\text { (cUS\$//b.) }\end{array}$ & Año & $\begin{array}{c}\text { Precio promedio anual } \\
\text { (cUS\$/lb.) }\end{array}$ \\
\hline 1987 & 90,94 & 1998 & 75,03 \\
\hline 1988 & 180,06 & 1999 & 71,33 \\
\hline 1989 & 128,71 & 2000 & 82,30 \\
\hline 1990 & 120,76 & 2001 & 71,60 \\
\hline 1991 & 106.06 & 2002 & 70,65 \\
\hline 1992 & 103,49 & 2003 & 80,73 \\
\hline 1993 & 87,98 & 2004 & 130,11 \\
\hline 1994 & 104,68 & 2005 & 167,09 \\
\hline 1995 & 133,14 & 2006 & 306,20 \\
\hline 1996 & 104,11 & 2007 & 322,91 \\
\hline 1997 & 103,27 & 2008 & 315,32 \\
\hline
\end{tabular}

Fuente: Banco Central de Chile.

Tabla 10. Índice de costos totales unitarios de cobre en Chile. * Empresas GMP-10** y CODELCO*** (Base real $2005=100)^{* * * * *}$

\begin{tabular}{|l|c|c|c|c|}
\hline \multicolumn{1}{|c|}{ Empresa /Año } & $\mathbf{2 0 0 5}$ & $\mathbf{2 0 0 6}$ & $\mathbf{2 0 0 7}$ & $\mathbf{2 0 0 8}$ \\
\hline Codelco & 100 & 117,0 & 136,4 & 167,2 \\
\hline Escondida & 100 & 124,5 & 130,9 & 198,4 \\
\hline Collahuasi & 100 & 112,9 & 102,8 & 118,4 \\
\hline Los Pelambres & 100 & 112,6 & 138,9 & 166,3 \\
\hline Anglo American Sur & 100 & 111,0 & 113,6 & 134,0 \\
\hline Candelaria & 100 & 95,0 & 106,9 & 116,8 \\
\hline El Abra & 100 & 97,9 & 120,2 & 164,7 \\
\hline Anglo American Norte & 100 & 102,9 & 105,9 & 146,6 \\
\hline Zaldívar & 100 & 92,6 & 102,9 & 128,6 \\
\hline Cerro Colorado & 100 & 86,3 & 112,9 & 118,6 \\
\hline Quebrada Blanca & 100 & 123,0 & 127,4 & 150,8 \\
\hline Variación anual & $7,7 \%$ & $13,1 \%$ & $8,6 \%$ & $27,1 \%$ \\
\hline
\end{tabular}

Fuente: Comisión Chilena del Cobre (Cochilco).

* Costos y gastos de la producción vendida (costos de explotación, gastos de administración y ventas, otros gastos operacionales, gastos financieros y otros no operacionales). Costos calculados sobre la base de la producción de cobre fino. ** GMP-10: son las 10 principales empresas productoras de cobre en Chile con contrato de inversión extranjera vía DFL-600 600 (Decreto con fuerza de ley, que regula el ingreso y salida de capitales extranjeros).

*** CODELCO (Corporación Chilena del Cobre) es la empresa de mayor producción de cobre fino en el mundo, de propiedad del Estado de Chile.

**** Deflactor: Producer Price Index de Estados Unidos (IPP, all Commodities). 
Tabla 11. Resumen de los proyectos mineros de cobre en el mundo.

\begin{tabular}{|c|c|c|c|c|c|c|c|c|c|}
\hline \multirow[b]{2}{*}{ Área geográfica } & \multicolumn{5}{|c|}{ Tipos de proyectos } & \multirow{2}{*}{$\begin{array}{c}\text { Reservas } \\
\text { asociadas } \\
\text { (MTM* } \\
\text { Mineral) }\end{array}$} & \multirow{2}{*}{$\begin{array}{l}\text { Ley de } \\
\text { Cobre } \\
(\%)^{* *}\end{array}$} & \multirow{2}{*}{$\begin{array}{l}\text { Inversión } \\
\text { Esperada } \\
(\text { US\$)*** }\end{array}$} & \multirow{2}{*}{$\begin{array}{l}\text { Producción } \\
\text { Nueva } \\
(\mathrm{TMF})^{* * * *}\end{array}$} \\
\hline & Total & $\begin{array}{l}\text { Green- } \\
\text { field }\end{array}$ & $\begin{array}{l}\text { Brown- } \\
\text { field }\end{array}$ & SxEw & $\begin{array}{c}\text { Concen- } \\
\text { trado }\end{array}$ & & & & \\
\hline África & 24 & 18 & 6 & 12 & 12 & $1.515,50$ & 1,84 & $4.710,00$ & 1.599 .000 \\
\hline Participación \% & & & & & & 2,74 & & 7,24 & 13,07 \\
\hline Latinoamérica & 69 & 57 & 12 & 29 & 40 & $32.474,23$ & 0,67 & $36.132,00$ & 6.693 .000 \\
\hline Participación \% & & & & & & 58,81 & & 55,55 & 54,72 \\
\hline Norteamérica & 26 & 22 & 4 & 7 & 19 & $5.145,60$ & 0,49 & $5.158,90$ & 936.000 \\
\hline Participación \% & & & & & & 9,32 & & 7,93 & 7,65 \\
\hline América & 95 & 79 & 16 & 36 & 59 & $37.619,83$ & 0,64 & $41.290,90$ & 7.629 .000 \\
\hline Participación \% & & & & & & 68,13 & & 63,48 & 62,37 \\
\hline Asia & 25 & 24 & 1 & 3 & 22 & $11.363,15$ & 0,65 & $9.707,50$ & 1.796 .000 \\
\hline Participación \% & & & & & & 20,56 & & 14,92 & 14,68 \\
\hline Europa & 7 & 6 & 1 & 1 & 6 & 856,10 & 0,54 & $1.936,00$ & 284.000 \\
\hline Participación \% & & & & & & 1,55 & & 2,98 & 2,33 \\
\hline Oceanía & 15 & 12 & 3 & 3 & 12 & $3.877,14$ & 0,94 & $7.398,60$ & 923.000 \\
\hline Participación \% & & & & & & 7,02 & & 11,38 & 7,55 \\
\hline \multirow[t]{2}{*}{ Total mundial } & 166 & 139 & 27 & 55 & 111 & $55.221,72$ & 0,70 & $65.042,90$ & 12.231 .000 \\
\hline & & & & & & 100,00 & & 100,00 & 100,00 \\
\hline
\end{tabular}

Fuente: Catastro mundial de proyectos y prospectos mineros de cobre, periodo 2006-2015, Cochilco (Aldo Picozzi B., Octubre-2007).

* $\quad$ Miles de toneladas métricas.

** Porcentaje de cobre contenido en cada tonelada de mineral procesado.

*** Millones de dólares de EE.UU.

**** Toneladas métricas anuales de cobre fino.

Tabla 12. Resumen de prospectos mineros de cobre en el mundo.

\begin{tabular}{|l|c|c|c|}
\hline \multicolumn{1}{|c|}{ Área geográfica } & $\mathbf{N}^{\circ}$ de prospectos & $\begin{array}{c}\text { Participación mundial } \\
(\boldsymbol{\%})\end{array}$ & $\begin{array}{c}\text { Ley estimada de cobre } \\
(\boldsymbol{\%})\end{array}$ \\
\hline África & 8 & 7,69 & $3 \%$ a $10 \%$ \\
\hline América & 68 & 65,39 & $0,3 \%$ a 5\% \\
\hline Latinoamérica & 53 & 50,96 & $0,5 \%$ a 3,96\% \\
\hline Norteamérica & 15 & 14,43 & $0,3 \%$ a 5\% \\
\hline Asia & 13 & 12,50 & $0,3 \%$ a 4\% \\
\hline Europa & 2 & 1,92 & $1,4 \%$ \\
\hline Oceanía & 13 & 12,50 & $0,4 \%$ a $1,5 \%$ \\
\hline Total Mundial & $\mathbf{1 0 4}$ & $\mathbf{1 0 0 , 0 0}$ & $\mathbf{0 , 3 \%}$ a 10\% \\
\hline
\end{tabular}

Fuente: Catastro mundial de proyectos y prospectos mineros de cobre, periodo 2006-2015, Cochilco (Aldo Picozzi B., Octubre-2007).

Tabla 11 muestra un resumen de los proyectos mineros de cobre en el mundo.

Respecto de los 104 prospectos mineros detectados, es decir, aquellos que podrían derivar en nuevos proyectos cupríferos en el mundo, 68 de ellos se ubican en América con una participación superior al $65 \%$ y dentro de ella, Latinoamérica con 53 prospectos y una participación de $78 \%$ en el continente y $51 \%$ a nivel mundial. La Tabla 12 muestra un resumen de los prospectos mineros de cobre debidamente ordenados por área geográfica. 


\section{CONCLUSIONES}

La producción y exportación del cobre está teniendo un rol fundamental para el crecimiento de algunas economías. Chile como principal país productor y exportador es un buen ejemplo de la importancia que reviste el metal rojo en el contexto de los aportes que su venta hace al PIB y con ello al bienestar de las personas y crecimiento de otras actividades que giran en torno a la producción y comercialización de este commodity.

Mediante la exposición de los principales antecedentes del mercado del cobre mundial se puede apreciar la importancia creciente que el cobre ha venido sosteniendo para los países que requieren de este metal para su industrialización, tal es el caso de China.

La reciente crisis financiera ha evidenciado la importancia que la comercialización del metal ha tenido para asegurar la estabilidad y evitar el desplome de economías emergentes como el caso de Chile al igual que otros países productores del cobre.

\section{REFERENCIAS}

[1] P. Jorion. "Valor en Riesgo: El nuevo paradigma para el control de riesgos con derivados". Editorial Limusa. México. 2000.

[2] P. Jorion. "Risk management lessons from Long-Term Capital Management". European
Financial Management. Vol. 6, pp. 277-300. Septiembre, 2000.

[3] C. Muñoz y C. Valencia. "Aspectos financieros relevantes de las empresas productoras de cobre internacionales". Comisión Chilena del Cobre. 2004.

[4] P. Crowson. "Mining in the global Market". 2001.

[5] Consejo Minero. "Informe de la Gran Minería Chilena”. Santiago, Chile. 2003.

[6] S. Lehmann, D. Moreno y P. Jaramillo. "Irrupción de China en la economía mundial: Efectos sobre el precio de commodities y desempeño de América Latina". Seminario los efectos de China en América. Banco Central de Chile. Abril, 2007.

[7] Diario Financiero. "Compendio Estadístico". Minería y Energía. N2 2. Junio 2006.

[8] COCHILCO. "Informe del mercado del cobre. Análisis 2008 y perspectivas 20092010”. Comisión Chilena del Cobre. 2008.

[9] J.C. Ciudad. "Determinantes del precio spot del cobre en las bolsas de metales". Recursos Naturales e Infraestructura. Serie 84. Cepal-Cochilco. Santiago, Chile. 2005.

[10] J. Cantallopts, R. Molina y O. Pérez. "Análisis histórico y estimaciones futuras del aporte de la minería al desarrollo de la economía chilena". COCHILCO. Documento de trabajo 28/3. Marzo 2008.

[11] A. Picozzi. "Estudio de COCHILCO". 2007. 